\title{
Huoxue Qianyang Qutan recipe attenuates Ang II-induced cardiomyocyte hypertrophy by regulating reactive oxygen species production
}

\author{
MINGTAI GUI*, LEI YAO*, BO LU, JING WANG, XUNJIE ZHOU, \\ JIANHUA LI, ZHENHUA DONG and DEYU FU
}

\begin{abstract}
Department of Cardiology, Yueyang Hospital of Integrated Traditional Chinese and Western Medicine, Shanghai University of Traditional Chinese Medicine, Shanghai 200437, P.R. China
\end{abstract}

Received April 9, 2021; Accepted September 15, 2021

DOI: $10.3892 /$ etm.2021.10881

\begin{abstract}
Continuous and irreversible cardiac hypertrophy can induce cardiac maladaptation and cardiac remodeling, resulting in increased risk of developing cardiovascular diseases. The present study was conducted to investigate the therapeutic effect of Huoxue Qianyang Qutan recipe (HQQR) on angiotensin II (Ang II)-induced cardiomyocyte hypertrophy. Primary cardiomyocytes were isolated from the cardiac tissue of neonatal rats, followed by flow cytometry detection to confirm the proportion of primary cardiomyocytes. Cell Counting Kit- 8 assay and immunofluorescence detection were performed to examine the effect of Ang II and HQQR on cardiomyocyte hypertrophy. Reactive oxygen species (ROS) and a series of metabolic indicators were quantified to investigate the effect of HQQR on Ang II-induced cardiomyocyte hypertrophy. Mitochondrial electron transport chain complex activity and related coding gene expression were determined to explore the effect of HQQR on mitochondrial function. HQQR significantly inhibited Ang II-induced cardiomyocyte hypertrophy and restored Ang II-induced ROS accumulation, metabolic indicators, and membrane potential levels. HQQR also regulated the mitochondrial function related to the sirtuin 1 pathway in Ang II-induced cardiomyocytes by increasing the activity of the mitochondrial electron transport chain complex and affecting the expression of genes encoding mitochondrial electron transport chain complex subunits. HQQR
\end{abstract}

Correspondence to: Dr Deyu Fu, Department of Cardiology, Yueyang Hospital of Integrated Traditional Chinese and Western Medicine, Shanghai University of Traditional Chinese Medicine, 110 Ganhe Road, Hongkou, Shanghai 200437, P.R. China

E-mail: fdy650@126.com

*Contributed equally

Key words: cardiomyocyte hypertrophy, Huoxue Qianyang Qutan recipe, mitochondrial dysfunction, mitochondrial electron transport chain could alleviate Ang II-induced cardiomyocyte hypertrophy by modulating oxidative stress, accumulating ROS and increasing mitochondrial electron transport chain activity.

\section{Introduction}

Cardiovascular diseases (CVDs) are a group of heart and blood vessel disorders that cause $\sim 17.5$ million deaths each year and have become the leading cause of death worldwide (1). Cardiac hypertrophy $(\mathrm{CH})$ is an adaptive reaction of cardiomyocytes to pressure overload and neurohumoral and cytokine abnormalities (2), which are manifested by increased size of cardiomyocytes and contractile protein synthesis $(3,4)$. However, continuous pathological stress overload results in irreversible $\mathrm{CH}$ and induces cardiac maladaptation and cardiac remodeling (5), thereby increasing the risk of developing various CVDs such as arrhythmia, myocardial ischemia, heart failure and sudden death.

Accumulating studies have demonstrated that, in myocardial remodeling, the content of angiotensin II (Ang II) is directly proportional to the degree of myocardial hypertrophy (6-8). Ang II directly participates in the development of myocardial hypertrophy by generating reactive oxygen species (ROS), reducing membrane potential and inducing mitochondrial dysfunction (6-8). Mitochondria are critical in energy metabolism, signal transduction and cell proliferation of cardiomyocytes (9). Mitochondrial dysfunctions are the major cause of $\mathrm{CH}$, which include mitochondrial ROS increase, electron transport chain protein damage, mitochondrial DNA (mtDNA) damage and a decrease in metabolic capacity (10-12). Therefore, reducing the content of Ang II and maintaining mitochondrial function in cardiomyocytes are potential strategies for the treatment of pathological $\mathrm{CH}$.

Traditional Chinese medicine (TCM) has a history of $>2,000$ years. It is widely used in Eastern Asia to prevent or treat a multitude of diseases, including CVDs (13). Tanshinones, berberine, matrine/oxymatrine, qiliqiangxin and Radix puerariae are representative compounds that can prevent or alleviate pathological $\mathrm{CH}$ and cardiac remodeling (14-17). Huoxue Qianyang Qutan recipe (HQQR) is a TCM compound consisting of Salviae miltiorrhizae, Stone 
Cassia, Ligusticum chuanxiong, Uncaria angustifolia, mulberry parasite, hawthorn and corn whisker. A previous study demonstrated that, in rats with obesity and hypertension $(\mathrm{OBH}), \mathrm{HQQR}$ could alleviate mitochondrial dysfunction and left ventricular hypertrophy by modulating the sirtuin 1 (SIRT1)/peroxisome proliferator-activated receptor-gamma coactivator-1 $\alpha$ (PGC-1 $\alpha$ ) deacetylation pathway in vivo (18). Furthermore, one of our previous studies revealed that the TCM Huoxue Qianyang decoction synergized with the activating transcription factor $6 / \mathrm{CHOP}$ endoplasmic reticulum stress signaling pathway and improved heart remodeling in rats with $\mathrm{OBH}$ (19). However, the therapeutic effect and the specific molecular mechanisms underlying the action of HQQR in Ang II-induced cardiomyocyte hypertrophy remain unclear.

Therefore, the present study was conducted to investigate the effect of HQQR on Ang II-induced cardiomyocyte hypertrophy in isolated primary cardiomyocytes, and to evaluate the role of HQQR in mitochondrial function to explore its underlying mechanism.

\section{Materials and methods}

Animals and drugs. All experimental procedures involving animals were approved by the Institutional Animal Care and Use Committee at Yueyang Hospital of Integrated Traditional Chinese and Western Medicine Affiliated to Shanghai University of Traditional Chinese Medicine (approval no. 18922), according to the principles outlined in the National Institutes of Health Guidelines for Care and Use of Laboratory Animals.

Pregnant Sprague Dawley rats $(n=2$; female; 10 weeks old; 260-270 g) purchased from the Vital River Laboratory Animal Technology Co., Ltd. [animal license number, SCXK (Beijing)] were fed ad libitum in an animal facility room with a 12 -h light/dark cycle at a temperature of $20-22^{\circ} \mathrm{C}$, with $\sim 50 \%$ humidity. After pregnant rats gave birth, neonatal rats (within $24 \mathrm{~h}$ of birth; 5-6 g) were euthanized using $\mathrm{CO}_{2}$ inhalation at a flow rate of $1.2 \mathrm{l} / \mathrm{min}$, which displaces $30 \%$ of the cage volume per min, followed with further cervical dislocation.

Valsartan capsules (Novartis International AG) were dissolved in $100 \%$ DMSO as $10 \mathrm{mmol} / 1$ stock solutions and stored at $-80^{\circ} \mathrm{C}$ before use. The compound stock solution was diluted in cell culture medium (DMEM medium with $10 \%$ FBS and $1 \%$ double-antibiotic) to a final concentration of $10 \mu \mathrm{mol} / 1$ (20). HQQR, consisting of Salvia miltiorrhiza, Stone Cassia, Ligusticum chuanxiong, Uncaria angustifolia, corn whisker, mulberry parasite and hawthorn (5:10:3:5:10:5:5), was decocted and dried according to a common protocol (18).

Isolation and culture of rat primary cardiomyocytes. Primary cardiomyocytes were isolated from the cardiac tissue of neonatal rats (within $24 \mathrm{~h}$ of birth) as previously described (21). Briefly, the hearts of the neonatal rats were collected, and the atrium of each heart was put into 5-ml sterile centrifuge tubes to be fully cut into small pieces. After full digestion with $0.4 \%$ Collagenase IV (cat. no. C4-BIOC; Sigma-Aldrich; Merck KGaA) and $0.05 \%$ trypsin-EDTA (cat. no. T1300; Beijing Solarbio Science \& Technology Co.,
Ltd.) in $37^{\circ} \mathrm{C}$ constant temperature water bath for $20 \mathrm{~min}$, the cell suspension was centrifuged at room temperature for $10 \mathrm{~min}$ at $1,000 \mathrm{x}$ g. After multiple digestion, the cell suspensions were filtered through a 100-mesh filter and mixed, and the cells were cultured at $37^{\circ} \mathrm{C}$ in DMEM medium (cat. no. SH30243.01; Hyclone; Cytiva) with 10\% FBS (cat. no. 16000-044, Gibco; Thermo Fisher Scientific, Inc.) and $1 \%$ double-antibiotic (penicillin-streptomycin; cat. no. P1400; Beijing Solarbio Science \& Technology Co., Ltd.) in a $37^{\circ} \mathrm{C}$, $5 \% \mathrm{CO}_{2}$ incubator. Next, the isolated cells were subjected to flow cytometry detection of troponin I (troponin I, TnI; 1:50; cat. no. ab196384; Abcam) to identify the purity of primary cardiomyocytes. The morphology of cardiomyocytes was observed, and pictures were captured with a light microscope (magnification, x200).

Experimental procedure. Cardiomyocytes were divided into the following five groups ( $5 \times 10^{5}$ cells; 6 -well plate): i) Vehicle (solvent group, DMSO); ii) Ang II + vehicle; iii) Ang II + $0.2 \mathrm{mg} / \mathrm{ml} \mathrm{HQQR}$; iv) Ang II + $0.5 \mathrm{mg} / \mathrm{ml} \mathrm{HQQR;} \mathrm{and}$ v) Ang II + valsartan. Ang II (cat. no. 4474-91-3) was purchased from Beijing Solarbio Science \& Technology Co., Ltd., and the corresponding concentration ( $1 \mu \mathrm{mol} / \mathrm{l})$ was prepared according to the manufacturer's instructions. Ang II and HQQR were added at the same time.

Fluorescent immunostaining. Cell suspensions containing $1 \times 10^{5}$ primary rat cardiomyocytes were added to a 24 -well plate and treated with Ang II, Ang II + $0.2 \mathrm{mg} / \mathrm{ml} \mathrm{HQQR,} \mathrm{Ang}$ II $+0.5 \mathrm{mg} / \mathrm{ml} \mathrm{HQQR}$, Ang II + $10 \mu \mathrm{mol} / \mathrm{l}$ valsartan, or Ang II + ROS scavenger (1 mM; cat. no. S6205; Selleck Chemicals) for $24 \mathrm{~h}$. Then, slides containing experimental primary cardiomyocytes were harvested, fixed with $4 \%$ formaldehyde for $30 \mathrm{~min}$ at room temperature, and permeabilized with $0.5 \%$ Triton X-100 in PBS for $10 \mathrm{~min}$ at room temperature. After blocking with $1 \%$ bovine serum albumin (BSA; cat. no. A8010; Beijing Solarbio Science \& Technology Co., Ltd.) in PBS for $30 \mathrm{~min}$ at room temperature, the slides were incubated with anti- $\alpha$-actinin antibody (1:200; cat. no. ab68194; Abcam) and anti- $\alpha$-smooth muscle actin ( $\alpha$-SMA; 1:500; ab32575; Abcam) overnight at $4^{\circ} \mathrm{C}$ in a wet-box. Subsequently, the slides were incubated with Alexa Fluor 555-labeled donkey anti-rabbit IgG $(\mathrm{H}+\mathrm{L})$ antibody (1:500; cat. no. A0453; Beyotime Institute of Biotechnology) for $1 \mathrm{~h}$ at room temperature in a wet-box before staining the nuclei with DAPI-containing media for $15 \mathrm{~min}$ at room temperature (1:500; cat. no. C1002; Beyotime Institute of Biotechnology). Pictures were taken using a fluorescence microscope (Nikon Corporation).

Cell Counting Kit-8 (CCK-8) assay. CCK-8 assay was performed in accordance with the manufacturer's kit protocol (Beyotime Institute of Biotechnology). Cell suspensions containing $3 \times 10^{3}$ primary rat cardiomyocytes were added to a 96-well plate. The cells were treated with $1 \mu \mathrm{mol} / 1$ Ang II (22) and gradient concentrations of $\mathrm{HQQR}(0,0.05,0.1,0.2,0.5$ and $1 \mathrm{mg} / \mathrm{ml})$ or valsartan $(10 \mu \mathrm{mol} / \mathrm{l})$ after $24 \mathrm{~h}$ of incubation. Finally, $10 \mu \mathrm{l}$ CCK-8 solution were added into each well, and the cells were incubated in an incubator at $37^{\circ} \mathrm{C}$ with $5 \% \mathrm{CO}_{2}$ for $1 \mathrm{~h}$. Cell proliferation at $0,12,24$ and $48 \mathrm{~h}$ was detected by recording the optical density at $450 \mathrm{~nm}\left(\mathrm{OD}_{450}\right)$. 
Table I. Primers for reverse transcription-quantitative PCR analysis.

\begin{tabular}{lll}
\hline Gene & \multicolumn{1}{c}{ Forward primer $\left(5^{\prime}\right.$ to $\left.3^{\prime}\right)$} & Reverse Primer (5' to $\left.3^{\prime}\right)$ \\
\hline ANP & GGGCTTCTTCCTCTTCCTG & TCTGAGACGGGTTGACTTCC \\
BNP & TAGCCAGTCTCCAGAACAATCC & ACCTCAGCCCGTCACAGC \\
$\beta$-MHC & TGACAACGCCTATCAGTACATG & CCTGGGGTCTGGTCCTTC \\
Nrf1 & GGTTAGGTGGCGAGTATGC & TATGAAGAGGTGTTGGTGGC \\
Tfam & ACCCAAGCATTACGGACC & CAGTACCAACCTGGATGAGC \\
Ndufa13 & CGCATACCCTCGCCTGTC & GTTCTGAAACTTTTGCATCTGG \\
SDHB & CGGGGACTGTCGGGATAC & GGAGGAGTGGCATGAGGG \\
COX IV & TACAAATCCATTGAGCCCTATC & GCACTCATACAATCCGTCCAG \\
COX1 & GGCGTGACTACCCCTTGC & CTCATTGGTGCCCTTGTTC \\
ATPase 6 & AACTAGGACAACCAGGAGCAC & AATCATTAGCGGCACAAGC \\
Ppargc1a & GAGCCGTAATTCTAGGTTCC & ATTGTAGCGGTTGGTGGG \\
mtDNA & AACCGCAGTCGCAACATG & GGAGGAGTCGTGGGAGGAG \\
GAPDH & AGCTCCAGCTTTTGTTCCC & CTTCCGGTTCGTATGTTGTG \\
\hline
\end{tabular}

ANP, atrial natriuretic peptide; BNP, brain natriuretic peptide; $\beta$-MHC, $\beta$-myosin heavy chain; Ppargc1a, peroxisome proliferator-activated receptor-gamma coactivator-1 $\alpha$; Nrf1, nuclear respiratory factor 1; Tfam, mitochondrial transcription factor A; Ndufa13, NADH:ubiquinone oxidoreductase subunit A13; SDHB, succinate dehydrogenase complex iron sulfur subunit B; COX IV, cytochrome $c$ oxidase subunit IV; COX1, anti-cyclooxygenase 1; Sirt1, sirtuin 1; mtDNA, mitochondrial DNA.

Reverse transcription-quantitative PCR. Total RNA was extracted from primary rat cardiomyocytes using TRIzol ${ }^{\circledR}$ reagent (cat. no. 15596026; Invitrogen; Thermo Fisher Scientific, Inc.) according to the manufacturer's instructions. Complementary DNA was synthesized using the RevertAid First Strand cDNA Synthesis kit (Fermentas; Thermo Fisher Scientific, Inc.) according to the manufacturer's instructions and under the following conditions: $37^{\circ} \mathrm{C}$ for $60 \mathrm{~min} ; 85^{\circ} \mathrm{C}$ for $5 \mathrm{~min} ; 4^{\circ} \mathrm{C}$ for $5 \mathrm{~min}$. mRNA levels were quantified using SYBR Green qPCR Master Mix (Thermo Fisher Scientific, Inc.) according to the manufacturer's instructions and under the following thermocycling conditions: $95^{\circ} \mathrm{C}$ for $10 \mathrm{~min}$; 40 cycles of $95^{\circ} \mathrm{C}$ for $15 \mathrm{sec}$ and $60^{\circ} \mathrm{C}$ for $45 \mathrm{sec}$. Relative mRNA levels were obtained using the $2^{-\Delta \Delta \mathrm{Cq}}$ method (23). GAPDH was used as an internal control for normalization. The primer sequences are listed in Table I.

Western blot analysis. The total protein of primary rat cardiomyocytes was extracted using RIPA buffer, which contained a protease and phosphatase inhibitor (cat. no. R0010; Beijing Solarbio Science \& Technology Co., Ltd.). Protein was then quantified using a BCA kit (cat. no. PICPI23223; Thermo Fisher Scientific, Inc.). Subsequently, proteins ( $25 \mu \mathrm{g}$ per lane) were separated using $10 \%$ SDS-PAGE and transferred to PVDF membranes. After blocking in 5\% non-fat milk for $1 \mathrm{~h}$ at room temperature, the protein bands were incubated with primary antibodies overnight at $4^{\circ} \mathrm{C}$, followed by incubation with the corresponding secondary antibodies [anti-rabbit (cat. no. A0208; 1:1,000; Beyotime Institute of Biotechnology) or anti-mouse (cat. no. A0216; 1:1,000; Beyotime Institute of Biotechnology) labeled with $\mathrm{HRP}]$ at $37^{\circ} \mathrm{C}$ for $1 \mathrm{~h}$. After a 5-min incubation with Immobilon Western chemiluminescent HRP substrate (cat. no. WBKLS0100; MilliporeSigma) in the dark, the protein bands were visually measured using a chemiluminescent imaging system (Tanon-5200, Tanon Science and Technology Co., Ltd.), and then quantified using ImageJ software (v1.8.0.112; National Institutes of Health). Anti-SIRT1 (cat. no. ab110304; 1:1,000), anti-PGC-1 $\alpha$ (cat. no. ab54481; 1:2,000), anti-nuclear respiratory factor 1 (NRF1; cat. no. ab175932; 1:5,000), anti-mitochondrial transcription factor A (Tfam; cat. no. ab131607; 1:2,000), anti-NADH:ubiquinone oxidoreductase subunit A13 (NDUFA13; cat. no. ab110240; 1:1,000), anti-succinate dehydrogenase complex iron sulfur subunit B (SDHB; cat. no. ab178423; 1:5,000), anti-cytochrome $c$ oxidase subunit IV (COX IV; cat. no. ab16056; 1:500), anti-cyclooxygenase 1 (COX1; cat. no. ab695; 1:1,000), anti-ATPase 6 (cat. no. ab192423; 1:500), anti-brain natriuretic peptide (BNP; cat. no. ab239510; 1:2,000), anti-Bax (cat. no. ab32503; 1:5,000), anti-Bcl-2 (cat. no. ab196495; 1:1,000) antibodies were obtained from Abcam, anti-cleaved caspase 3 (cat. no. 9661; 1:1,000), anti-caspase 3 (cat. no. 9662; 1:1,000) were obtained from CST, anti-atrial natriuretic peptide (ANP; cat. no. RQ4453; $0.5 \mu \mathrm{g} / \mathrm{ml}$ ) antibody was obtained from NSJ Bioreagents, anti- $\beta$-myosin heavy chain $(\beta-\mathrm{MHC}$; cat. no. MA1-26180; $0.5 \mu \mathrm{g} / \mathrm{ml}$ ) antibody was obtained from Invitrogen; Thermo Fisher Scientific, Inc., and anti-GAPDH as a loading control (cat. no. 60004-1-1G; 1:5,000) was obtained from ProteinTech Group, Inc. The antibodies were diluted in PBST (containing $0.05 \%$ Tween-20).

ROS detection. ROS accumulation in cardiomyocytes was quantified using the Active Oxygen Detection kit (cat. no. S0033; Beyotime Institute of Biotechnology) according to the manufacturer's instructions. After treatment with $1 \mu \mathrm{mol} / \mathrm{l} \mathrm{Ang} \mathrm{II} \mathrm{and}$ HQQR $(0.2$ and $0.5 \mathrm{mg} / \mathrm{ml})$ or valsartan $(10 \mu \mathrm{mol} / \mathrm{l})$, DCFH-DA probe solution was prepared and used for incubation with the harvested cell samples at $37^{\circ} \mathrm{C}$ for $30 \mathrm{~min}$ in the dark. The cells 
A

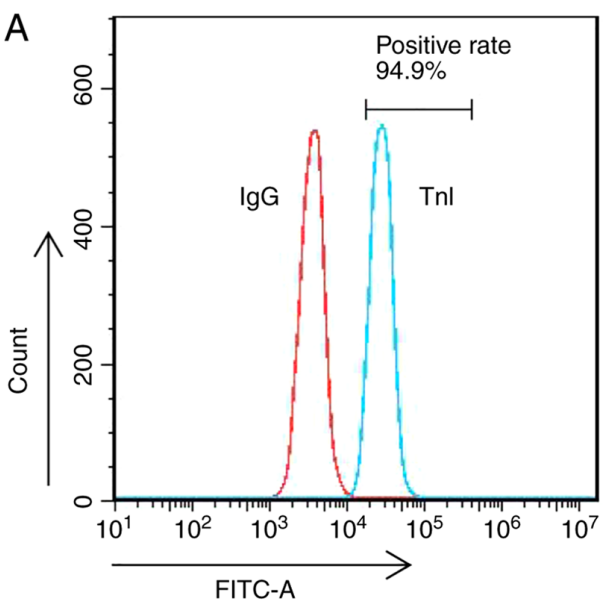

B

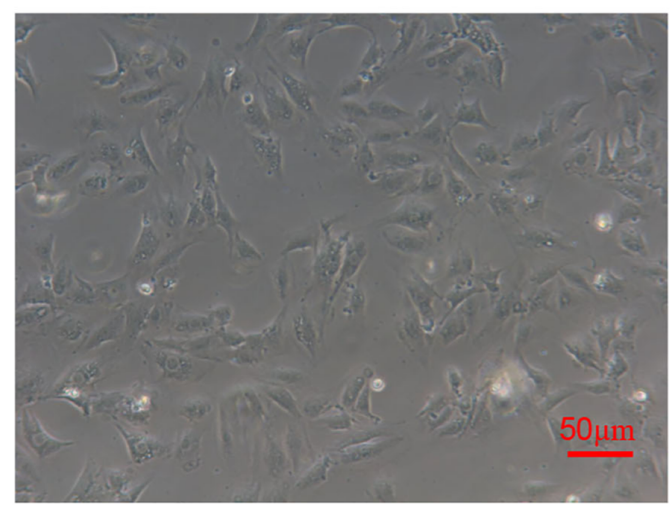

C

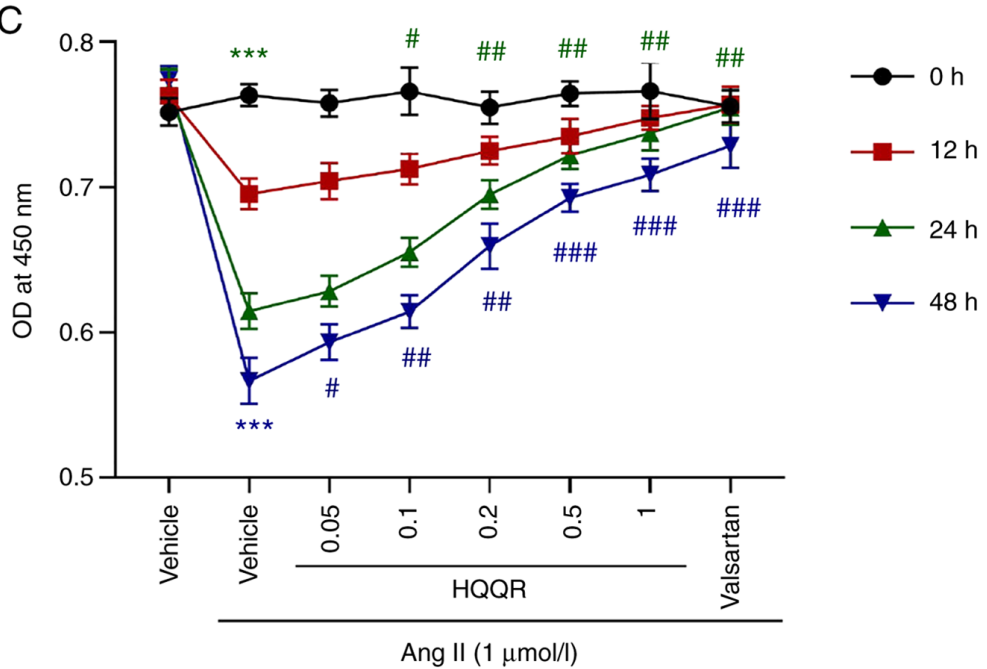

Figure 1. HQQR attenuates the inhibitory effect of Ang II on rat cardiomyocyte proliferation. (A) TnI was detected using flow cytometry analysis to identify the percentage of primary cardiomyocytes. (B) Image of isolated primary rat cardiomyocytes (magnification, x200; scale bar, $50 \mu \mathrm{m}$ ). (C) Cell Counting Kit- 8 assays were used to examine the proliferation of primary cardiomyocytes at $0,12,24$ and $48 \mathrm{~h}$ after treatment with Ang II ( $1 \mu$ mol/l) and HQQR powder $(0,0.05,0.1,0.2,0.5$ and $1 \mathrm{mg} / \mathrm{ml})$. Vehicle and valsartan were used as negative and positive controls, respectively. ${ }^{* * * *} \mathrm{P}<0.001 \mathrm{vs}$. the vehicle group; ${ }^{*} \mathrm{P}<0.05$, ${ }^{\# \prime} \mathrm{P}<0.01$ and ${ }^{\# \# \#} \mathrm{P}<0.001$ vs. the Ang II + vehicle group. HQQR, Huoxue Qianyang Qutan recipe; Ang II, angiotensin II; TnI, troponin I; OD, optical density.

and solution were gently inverted every $10 \mathrm{~min}$. After washing three times with DMEM without serum, the samples were examined via flow cytometry (Accuri C6; BD Biosciences) using FlowJo software (v10.0.7r2; Tree Star, Inc.).

Biochemical detection. Primary rat cardiomyocytes were treated with $1 \mu \mathrm{mol} / 1$ Ang II and HQQR $(0.2$ and $0.5 \mathrm{mg} / \mathrm{ml})$ or valsartan $(10 \mu \mathrm{mol} / \mathrm{l})$ for $24 \mathrm{~h}$, and then, the cells were collected, and the supernatant was kept. The levels of ATP and protein carbonyl, and the activities of glutathione peroxidase (GSH-PX), catalase (CAT), malondialdehyde (MDA) and superoxide dismutase (SOD) were respectively detected using the BCA (cat. no.A045-3), ATP (cat.no. A095), protein carbonyl (cat.no.A087), GSH-PX (cat. no. A005), CAT (cat. no. A007-1), MDA (cat. no. A003-2) and SOD (cat. no. A001-1) kits (Nanjing Jiancheng Bioengineering Institute) according to the manufacturer's instructions, and measured using a visible spectrophotometer. The activities of the mitochondrial complexes I-IV and V were respectively detected using the mitochondrial complex I (cat. no. BC0515), II (cat. no. BC3235), III (cat. no. BC3245), IV (cat. no. BC0945) and V (cat. no. BC1445) Activity Test kits (Beijing
Solarbio Science \& Technology Co., Ltd.) and measured using a UV spectrophotometer.

Mitochondrial potential assay. Alterations in mitochondrial membrane potential were evaluated using the Mitochondrial Membrane Potential Detection kit with JC-1 (cat. no. C2006; Beyotime Institute of Biotechnology). According to the manufacturer's instructions, the cell suspension was prepared and detected using a flow cytometer (Accuri C6; BD Biosciences) via the software of Flow Jo (v10.0.7r2 version). JC-1 is an ideal fluorescent probe widely used to detect mitochondrial membrane potential. In high mitochondrial membrane potential, JC-1 aggregates in the matrix of mitochondria and forms polymer (J-aggregates), which produces red fluorescence. When the mitochondrial membrane potential is low, JC-1 cannot aggregate in the matrix of mitochondria. At this time, JC- 1 is a monomer and can produce green fluorescence.

Cell apoptosis. Primary rat cardiomyocyte cells after $24 \mathrm{~h}$ treatment with Ang II, Ang II + $0.2 \mathrm{mg} / \mathrm{ml} \mathrm{HQQR,} \mathrm{Ang} \mathrm{II} \mathrm{+}$ 
A

Vehicle

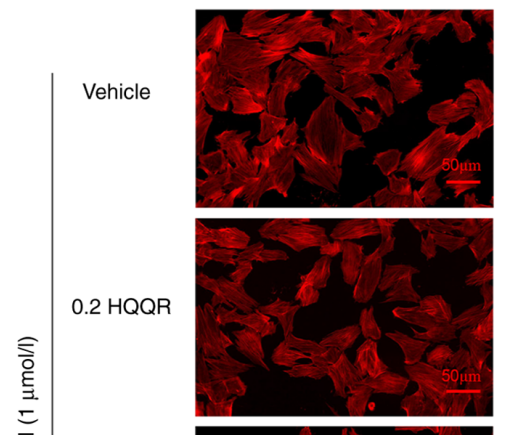

0.5 HQQR

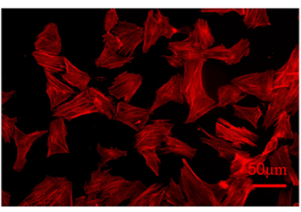

Valsartan

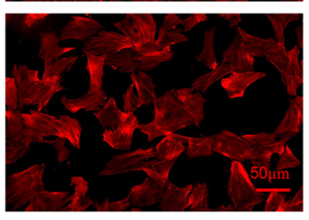

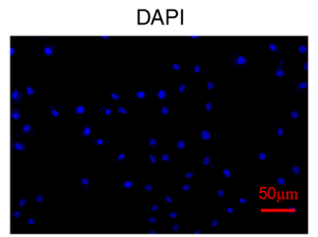
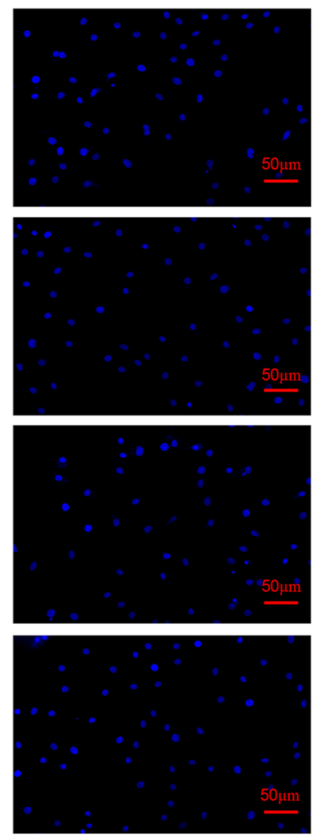
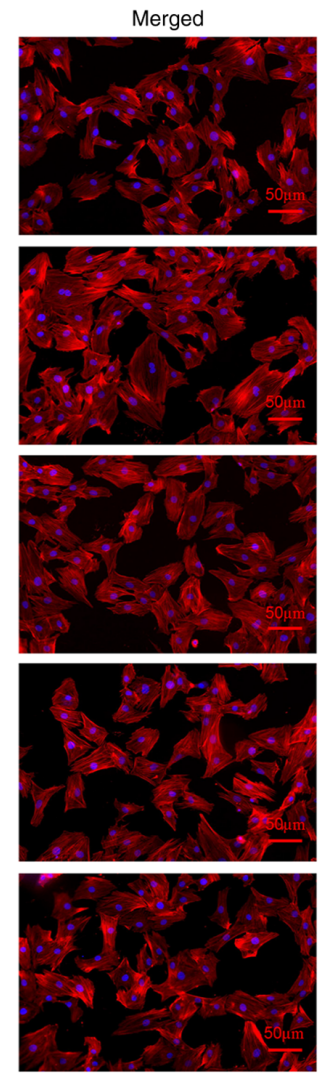

$\mathrm{B}$

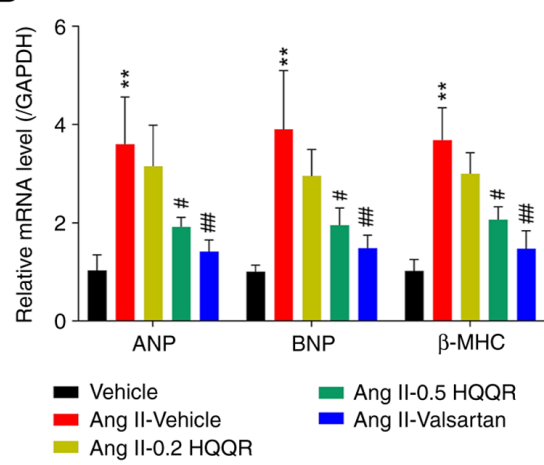

C

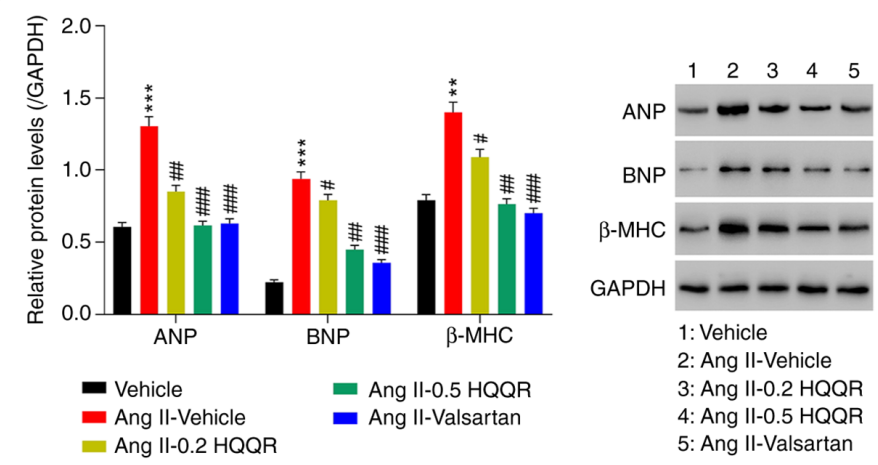

Figure 2. HQQR improves Ang II-induced cardiomyocyte hypertrophy. (A) Immunofluorescence experiments were performed using anti- $\alpha$-actinin antibodies to analyze cardiomyocyte hypertrophy (magnification, x200; scale bar, $50 \mu \mathrm{m}$ ). (B) mRNA expression levels of the myocardial hypertrophy markers ANP, BNP and $\beta$-MHC were examined using reverse transcription-quantitative PCR. (C) Protein expression levels of the myocardial hypertrophy markers ANP, BNP and $\beta$-MHC were examined using western blotting. ${ }^{* *} \mathrm{P}<0.01$ and ${ }^{* * *} \mathrm{P}<0.001$ vs. the vehicle group; ${ }^{\#} \mathrm{P}<0.05$, ${ }^{\# \#} \mathrm{P}<0.01$ and ${ }^{\# \# \#} \mathrm{P}<0.001$ vs. the Ang II + vehicle group. HQQR, Huoxue Qianyang Qutan recipe; Ang II, angiotensin II; ANP, atrial natriuretic peptide; BNP, brain natriuretic peptide; $\beta$-MHC, $\beta$-major histocompatibility complex.

$0.5 \mathrm{mg} / \mathrm{ml}$ HQQR, Ang II + $10 \mu \mathrm{mol} / 1$ valsartan were stained using Annexin V Apoptosis kit (C1062; Beyotime Institute of Biotechnology) according to the manufacturer's instructions and a Beckman CytoFLEX Flow Cytometer (Beckman Coulter, Inc.) with FlowJo software (v10.0.7r2; Tree Star, Inc.) was used to analyze apoptosis.

Statistical analysis. Statistical analysis was conducted using GraphPad Prism 7.0 software (GraphPad Software, Inc.). Data are presented as the mean \pm SD. One-way ANOVA followed by Tukey's post hoc test was applied for comparisons between groups. Results are representative of at least three repeated experiments. $\mathrm{P}<0.05$ was considered to indicate a statistically significant difference.

\section{Results}

HQQR attenuates the inhibitory effect of Ang II on rat cardiomyocyte proliferation. To investigate the effect of HQQR on Ang II-induced cardiomyocyte hypertrophy, rat primary cardiomyocytes were isolated. After detection using TnI, the results of flow cytometry analysis confirmed that $\sim 95 \%$ of the isolated cells were primary cardiomyocytes (Fig. 1A). Microscopic observations (light microscope) also indicated that the isolated cell morphology was consistent with the morphological characteristics of primary cardiomyocytes (Fig. 1B), including a short columnar form with generally only one nucleus being mostly located in the middle of the cell, while the cell shape is ellipse- or 
A

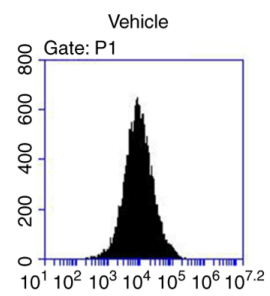

Gate: P1

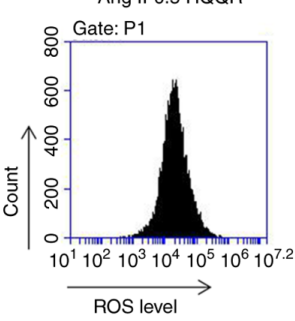

$\mathrm{F}$

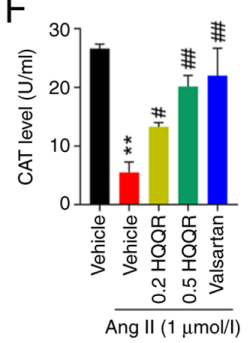

G

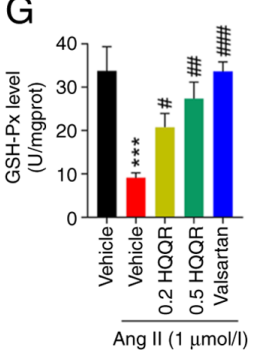

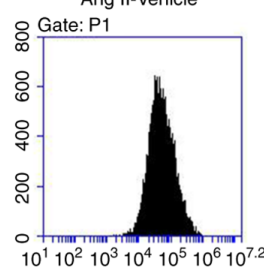

Ang II-Valsartan
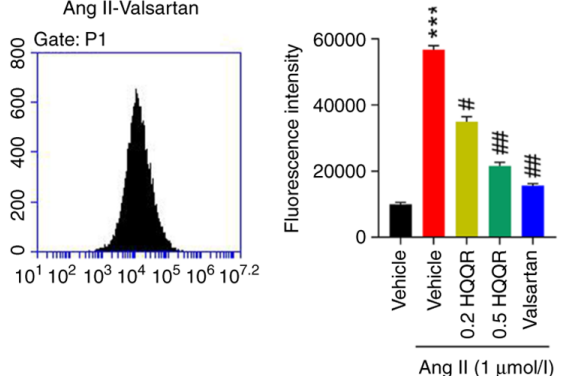

B
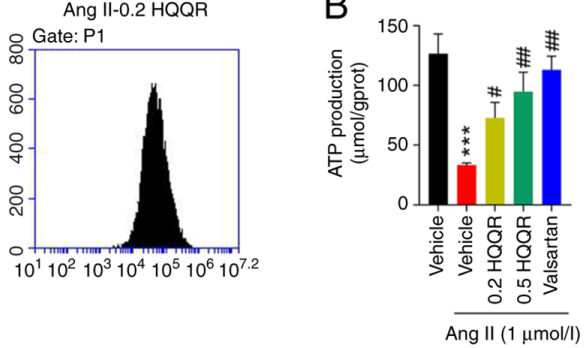

D

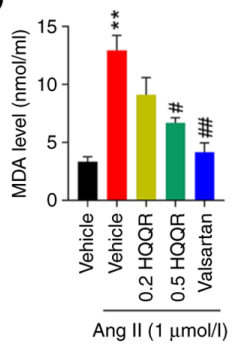

DAPI
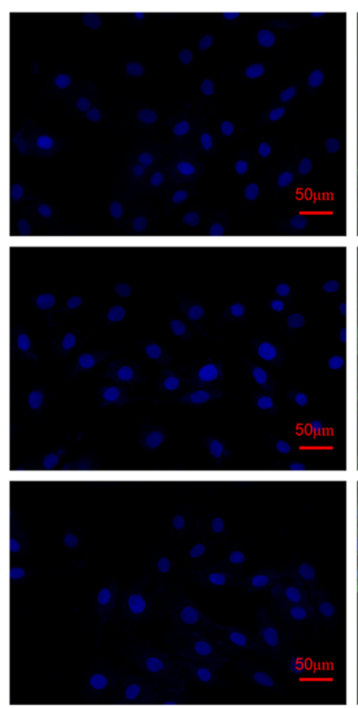

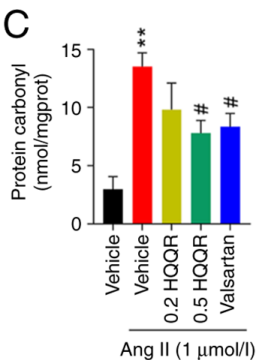

E

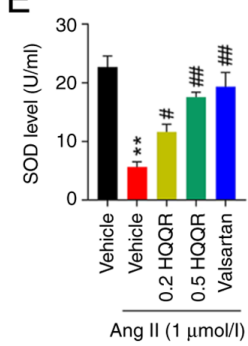

Merged
$\mathrm{H}$
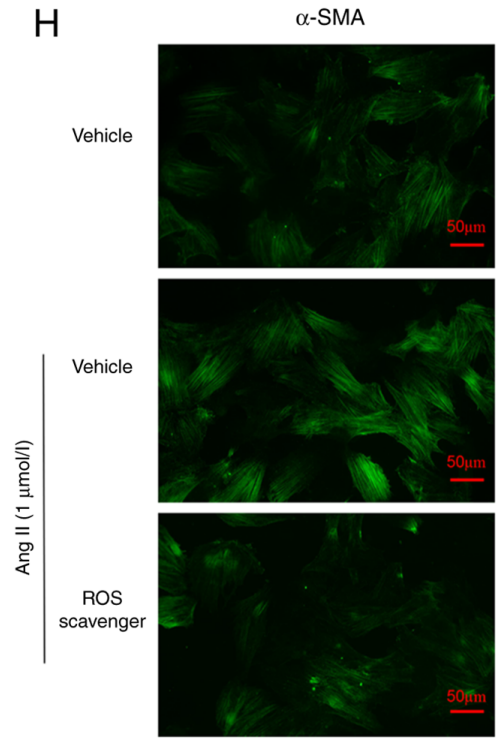

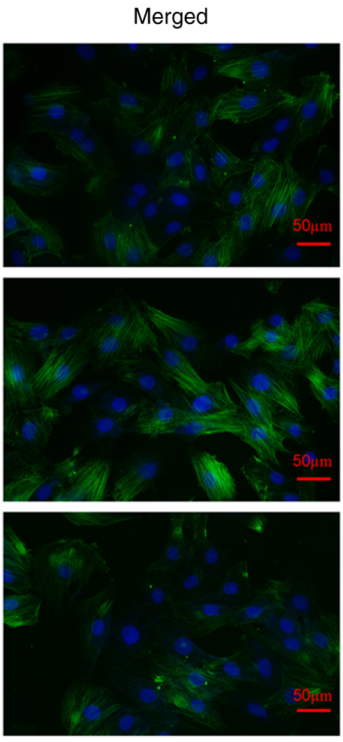

Figure 3. HQQR alleviates AngII-induced cardiomyocyte hypertrophy by modulating ROS accumulation. (A) Flow cytometry analysis to detect the changes in ROS accumulation. Levels of (B) ATP and (C) protein carbonyl content, and activities of (D) MDA, (E) SOD, (F) CAT and (G) GSH-Px were quantified. (H) Immunofluorescence experiments were performed using anti- $\alpha$-SMA antibodies to analyze cardiomyocyte hypertrophy (magnification, $x 200$; scale bar, $50 \mu \mathrm{m})$. ${ }^{* *} \mathrm{P}<0.01$ and ${ }^{* * *} \mathrm{P}<0.001$ vs. the vehicle group; $\mathrm{P}<0.05,{ }^{\# \#} \mathrm{P}<0.01$ and ${ }^{\# \# \#} \mathrm{P}<0.001$ vs. the Ang II + vehicle group. HQQR, Huoxue Qianyang Qutan recipe; Ang II, angiotensin II; MDA, malondialdehyde; SOD, superoxide dismutase; CAT, catalase; GSH-PX, glutathione peroxidase; $\alpha$-SMA, $\alpha$-smooth muscle actin.

rectangle-like, and its long axis is in the same direction as in myofibrils. The cytotoxicity of HQQR was detected using a CCK-8 assay (Fig. S1), the results of which revealed that 0.2 and $0.5 \mathrm{mg} / \mathrm{ml} \mathrm{HQQR} \mathrm{had} \mathrm{no} \mathrm{cytotoxicity} \mathrm{and} \mathrm{the} 24 \mathrm{~h}$ after treatment had the best effect. Cell proliferation experiments revealed that, compared with normally cultured (medium without other treatment) primary cardiomyocytes, Ang II (1 $\mu \mathrm{mol} / \mathrm{l})$ treatment significantly inhibited the proliferation of primary cardiomyocytes, which was markedly abolished by incubation with valsartan $(10 \mu \mathrm{mol} / \mathrm{l})$, the antagonist of angiotensin receptor used as a positive control. The addition of HQQR solution ameliorated the inhibitory effect of Ang II on primary cardiomyocyte proliferation in a dose-dependent manner (Fig. 1C). On the basis of the present results, Ang II treatment for $24 \mathrm{~h}$ followed by 0.2 and $0.5 \mathrm{mg} / \mathrm{ml} \mathrm{HQQR}$ concentrations were selected as treatment conditions for subsequent experiments. Valsartan was used as a positive control.

HQQR improves AngII-induced cardiomyocyte hypertrophy. To further examine the role of HQQR in Ang II-induced cardiomyocyte hypertrophy, primary cardiomyocytes were divided into the following five groups: i) Vehicle; ii) Ang II $(1 \mu \mathrm{mol} / 1)+$ vehicle; iii) Ang II $(1 \mu \mathrm{mol} / \mathrm{l})+\mathrm{HQQR}$ (0.2 mg/ml); iv) Ang II (1 $\mu \mathrm{mol} / \mathrm{l})+\operatorname{HQQR}(0.5 \mathrm{mg} / \mathrm{ml})$; and v) valsartan + Ang II (1 $\mu \mathrm{mol} / \mathrm{l})$. The immunofluorescence of $\alpha$-actinin revealed that, compared with that in the vehicle group, cardiomyocyte hypertrophy was observed in the Ang II-treated group, indicating that Ang II induced cardiomyocyte hypertrophy. However, treatment with HQQR could significantly inhibit Ang II-induced cardiomyocyte hypertrophy (Fig. 2A) and reduce the Ang II-induced expression of the 
A
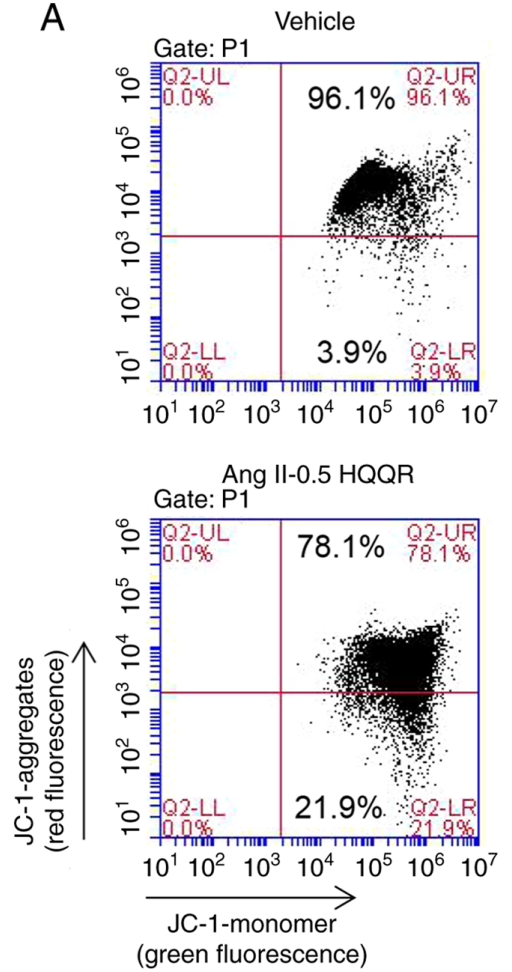

B
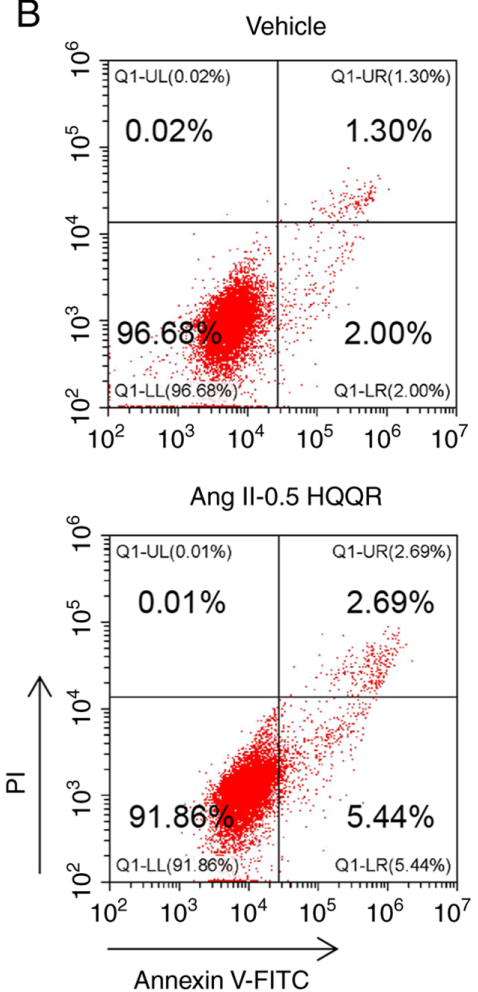
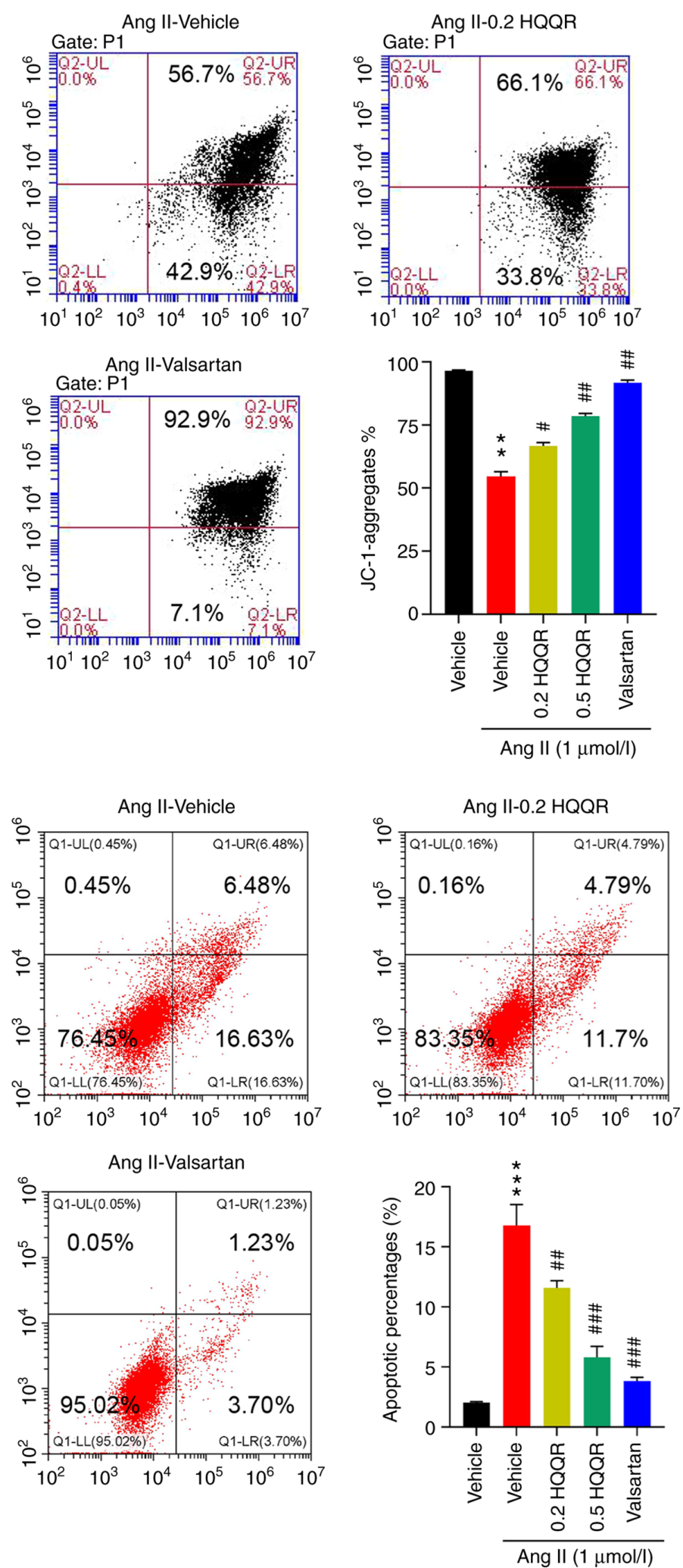

Figure 4. HQQR reverts cardiomyocyte hypertrophy-induced membrane potential reduction and apoptosis increase. (A) Flow cytometry analysis to detect the changes in membrane potential. (B) Flow cytometry analysis to detect the changes in cell apoptosis. ${ }^{* *} \mathrm{P}<0.01$ and ${ }^{* * *} \mathrm{P}<0.001$ vs. the vehicle group; ${ }^{*} \mathrm{P}<0.05$, ${ }^{\# \#} \mathrm{P}<0.01$ and ${ }^{\# \#} \mathrm{P}<0.001$ vs. the Ang II + vehicle group. HQQR, Huoxue Qianyang Qutan recipe; Ang II, angiotensin II.

myocardial hypertrophy markers ANP, BNP and $\beta$-MHC (Fig. 2B and C). Valsartan was used as a positive control.

HQQR alleviates AngII-induced cardiomyocyte hypertrophy by modulating ROS accumulation. Ang II treatment markedly enhanced ROS accumulation, whereas treatment with HQQR significantly inhibited ROS accumulation in Ang II-treated primary cardiomyocytes, in a dose-dependent manner (Fig. 3A). HQQR could also rescue the effect of Ang II treatment on the levels of MDA, SOD, ATP, CAT, 


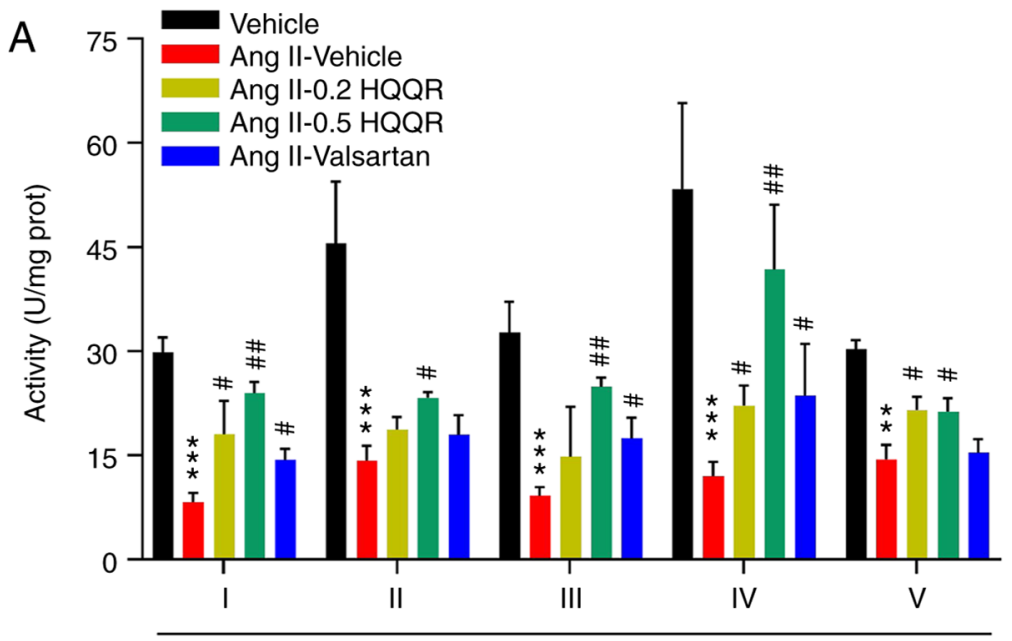

Mitochondrial complex
B

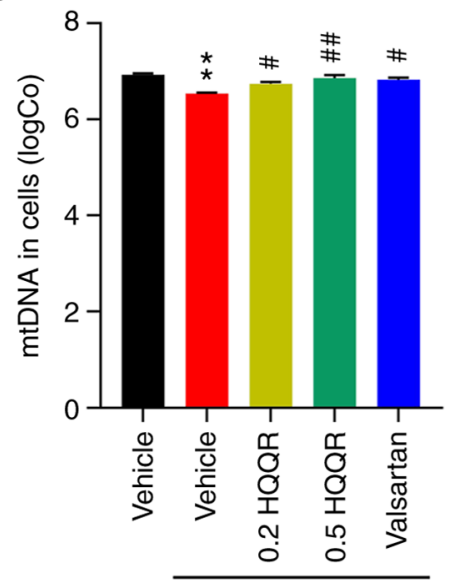

Ang II $(1 \mu \mathrm{mol} / \mathrm{l})$
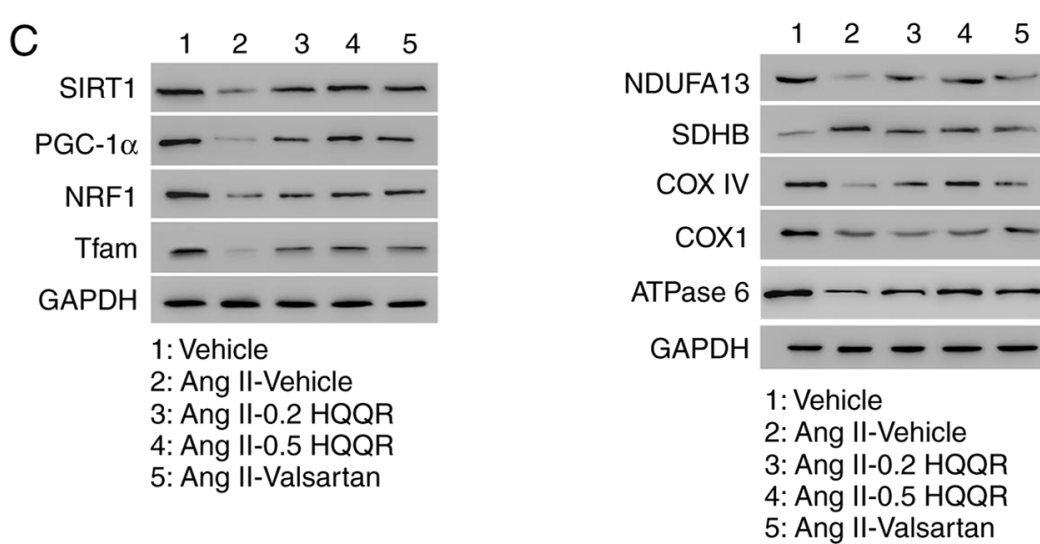

Figure 5. HQQR increases the activity of mitochondrial electron transport chain complexes in Ang II-treated rat cardiomyocytes. (A) The activities of the mitochondrial complexes I-IV and V were determined. (B) The level of mtDNA was quantified using RT-qPCR. (C) Protein levels of SIRT1, PGC-1 $\alpha$, NRF1, Tfam, NDUFA13, SDHB, COX IV, COX1 and ATPase 6 in primary cardiomyocytes were examined by western blotting. ${ }^{* *} \mathrm{P}<0.01$ and ${ }^{* * *} \mathrm{P}<0.001 \mathrm{vs}$. the vehicle group; ${ }^{\mathrm{P}}<0.05$ and ${ }^{\#} \mathrm{P}<0.01$ vs. the Ang II + vehicle group. HQQR, Huoxue Qianyang Qutan recipe; Ang II, angiotensin II; SIRT1, sirtuin 1; mtDNA, mitochondrial DNA; RT-qPCR, reverse transcription-quantitative PCR; PGC-1 $\alpha$, peroxisome proliferator-activated receptor-gamma coactivator-1 $\alpha$; NRF1, nuclear respiratory factor 1; Tfam, mitochondrial transcription factor A; NDUFA13, NADH:ubiquinone oxidoreductase subunit A13; SDHB, succinate dehydrogenase complex iron sulfur subunit B; COX IV, cytochrome $c$ oxidase subunit IV; COX1, anti-cyclooxygenase 1; RT-qPCR, reverse transcription-quantitative PCR.

GSH-Px and protein carbonyl in primary cardiomyocytes (Fig. 3B-G). Consistent with these results, the immunofluorescence of $\alpha$-SMA revealed that ROS was directly involved in AngII-induced cardiomyocyte hypertrophy, and that the ROS scavenger alleviated AngII-induced cardiomyocyte hypertrophy (Fig. 3H). The present findings suggested that HQQR treatment could alleviate Ang II-induced cardiomyocyte hypertrophy, potentially by modulating ROS accumulation. Valsartan was used as a positive control.

HQQR reverts cardiomyocyte hypertrophy-induced membrane potential reduction and apoptosis increase. Ang II treatment notably reduced mitochondrial membrane potential (Fig. 4A) and increased cell apoptosis (Fig. 4B). In Ang II-stimulated cardiomyocytes, treatment with HQQR significantly reversed the hypertrophy-induced membrane potential reduction and apoptosis in a dose-dependent manner. Moreover, Ang II induced the expression of apoptosis-related proteins Bax and cleaved caspase 3, which were significantly decreased upon HQQR treatment, while anti-apoptotic Bcl-2 was significantly decreased by Ang-II and increased by HQQR (Fig. S2). Valsartan was used as a positive control.

$H Q Q R$ increases the activity of mitochondrial electron transport chain complexes in Ang II-treated rat cardiomyocytes. To further explore the mechanism by which HQQR affects Ang II-induced cardiomyocyte hypertrophy, the activities of the mitochondrial complexes I-IV and $\mathrm{V}$ were studied $24 \mathrm{~h}$ after treatment. As presented in Fig. 5A, Ang II markedly inhibited the activity of the mitochondrial complexes I-IV and V, whereas the addition of HQQR or valsartan partially rescued their activities. Furthermore, HQQR or valsartan treatment markedly inhibited Ang II-induced mtDNA leakage (Fig. 5B). The mRNA and protein expressions of genes encoding the mitochondrial complex and involved in mitochondrial biogenesis were subsequently evaluated. As indicated in Figs. 5C and S3, Ang II treatment reduced both mRNA and protein levels of SIRT1, PGC-1 $\alpha$, NRF1, Tfam, NDUFA13, COX IV, COX1 and ATPase 6, and elevated the expression of SDHB. These effects were notably 
rescued by treatment with HQQR or valsartan, which was used as a positive control.

\section{Discussion}

$\mathrm{CH}$ is generally considered to be a major inducer of several types of CVDs (24). There is a need to explore effective therapeutic targets with low toxicity for the clinical treatment of $\mathrm{CH}$ and CVDs. Because of its low toxicity, TCM has been used to treat chronic diseases in East Asia throughout history. Several types of TCM have been demonstrated to be effective for $\mathrm{CH}$ treatment by affecting multiple signaling pathways or physiological activities. For instance, Bu-Shen-Jiang-Ya decoction suppressed left ventricular hypertrophy by regulating the extracellular signal-regulated kinase signaling pathway in spontaneously hypertensive rats (25). Moreover, QiShenYiQi pills ameliorated fatigue-induced $\mathrm{CH}$ through the regulation of energy metabolism (26). Qiliqiangxin was also found to attenuate phenylephrine-induced $\mathrm{CH}$ by upregulating peroxisome proliferator-activated receptor $\gamma$ and PGC-1 $\alpha$ (27). Combined with the role of $\operatorname{HQQR}(18,19)$ in hypertensive rats, these findings provide evidence that TCM or a combination therapy of TCM and western medicine has the potential to treat $\mathrm{CH}$. It is necessary to investigate TCM targeting $\mathrm{CH}$ treatment further, along with the potential mechanisms to prevent drug resistance and improve therapeutic effectiveness.

In the present study, the CCK-8 and immunofluorescence experiments confirmed that Ang II could induce CH (6). Functional analysis of $\mathrm{HQQR}$ in the treatment of $\mathrm{CH}$ revealed that HQQR significantly alleviated Ang II-induced $\mathrm{CH}$ in primary cardiomyocytes, thereby suggesting the significance of HQQR in $\mathrm{CH}$ treatment.

The mechanism by which HQQR abolished Ang II-induced $\mathrm{CH}$ was investigated. Mitochondria are double-membrane organelles that support a variety of physiological activities, including energy production, cell death and oxidative metabolism (9). Mitochondrial dysfunction has been implicated in the occurrence and development of cardiomyocyte hypertrophy (28). Mitochondrial biogenesis is essential for sustaining mitochondrial function and is related to ROS production (29). Although acute or mild oxidative stress can trigger mitochondrial biogenesis due to the involvement of mitochondrial quality control (30), the present study reported that Ang II treatment promoted the accumulation of ROS in cardiomyocytes and induced the disturbance of mitochondrial biogenesis, which may further result in cardiomyocyte injury and hypertrophy. HQQR treatment attenuated oxidative stress and restored the levels of mitochondrial transcription factors in cardiomyocytes. The inhibition of ROS accumulation and the normal activity of mitochondrial electron transport chain are crucial for alleviating mitochondrial-related diseases (10). After Ang II-induced ROS production, ROS activate several critical signaling pathways related to cell death and fibrosis, such as the mitogen-activated protein kinase pathway, the extracellular regulated protein kinase signaling pathway, protein kinase $\mathrm{C}$ signaling and NF- $\mathrm{NB}$; these signaling pathways participate in the process of $\mathrm{CH}$ (31-33). Therefore, drugs that directly or indirectly target ROS production might inhibit the occurrence or development of $\mathrm{CH}$. As anticipated, $\mathrm{HQQR}$ treatment significantly reduced the accumulation of ROS and the indicators of oxidative stress, and elevated the levels of scavenging system indicators, such as SOD, CAT and GSH-PX, thus suggesting that HQQR partially prevented Ang II-induced $\mathrm{CH}$ by reducing the accumulation of ROS and repressing oxidative stress in myocardial cells. Ang II reduced the activity of the mitochondrial electron transport chain and suppressed the expression of mitochondrial complex subunits in cardiomyocytes; however, this impact was significantly reverted by HQQR treatment, indicating that HQQR could also exert its therapeutic effect by maintaining normal electron transfer.

HQQR treatment increased the ATP content in cardiomyocytes and elevated the levels of NRF1 and Tfam, which are critical for mitochondrial biogenesis. SIRT1 is a deacetylase that plays a key role in cell proliferation, differentiation, senescence, apoptosis and metabolism (34). The SIRT1/PGC-1 $\alpha$ pathway contributes to mitochondrial dysfunction and mitochondrial biogenesis $(35,36)$. Inhibition of PGC- $1 \alpha$ or PGC-1 $\alpha$ deacetylation significantly abolishes the process leading to $\mathrm{CH}$ (27). The present study indicated that HQQR rescued Ang II-inhibited SIRT1 and PGC-1 $\alpha$ expression, thus implying that HQQR protected cardiomyocytes from the development of $\mathrm{CH}$ by enhancing the SIRT1/PGC-1 $\alpha$ regulatory pathway. It can be speculated that HQQR improves mitochondrial biosynthesis, leading to the reduction of oxidative stress.

Currently, there are relatively few studies on the efficacy of HQQR in CVDs. The present study provided evidence for the protective role of HQQR in $\mathrm{CH}$, enriching the strategies for $\mathrm{CH}$ treatment. Furthermore, our previous study (18) confirmed the effect of HQQR in glucolipid metabolism and blood pressure regulation, thereby demonstrating the potential of $\mathrm{HQQR}$ in treating metabolic diseases and CVDs.

However, the effect of HQQR on the development of $\mathrm{CH}$ was only investigated at the cellular level. Therefore, in vivo experiments are required to further confirm the therapeutic effect of HQQR on $\mathrm{CH}$ and further explore underlying molecular mechanisms to expand the clinical application of HQQR.

The present study provided evidence that Ang II treatment could induce $\mathrm{CH}$ in primary cardiomyocytes, and that HQQR could significantly prevent Ang II-induced $\mathrm{CH}$ by preventing mitochondrial dysfunction and oxidative stress, reducing ROS accumulation and protecting the electron transport chain. Although additional studies are required to further verify the function and mechanisms of HQQR in the development of $\mathrm{CH}$, the present study implied that HQQR could be considered a novel approach for $\mathrm{CH}$ treatment.

\section{Acknowledgements}

Not applicable.

\section{Funding}

The present study was funded by the National Natural Science Foundation of China (grant nos. 81774111 and 81803892) and Scientific research projects of Shanghai Science and Technology Commission (grant no. 19401970400). 


\section{Availability of data and materials}

All data generated or analyzed during this study are included in this published article.

\section{Authors' contributions}

DF conceived and designed the study. MG, LY, BL, JW, $\mathrm{XZ}, \mathrm{JL}$ and ZD performed the experiments. DF wrote the manuscript. DF and MG have confirmed the authenticity of all the raw data. All authors have read and approved the final manuscript.

\section{Ethics approval and consent to participate}

All the experiments conducted in the present study were approved by the Ethics Committee of Yueyang Hospital of Integrated Traditional Chinese and Western Medicine Affiliated to Shanghai University of Traditional Chinese Medicine (approval no. 18922, Shanghai, China).

\section{Patient consent for publication}

Not applicable.

\section{Competing interests}

The authors declare that they have no competing interests.

\section{References}

1. Badimon L, Chagas P and Chiva-Blanch G: Diet and cardiovascular disease: Effects of foods and nutrients in classical and emerging cardiovascular risk factors. Curr Med Chem 26 3639-3651, 2019.

2. Xu L, Su Y, Zhao Y, Sheng X, Tong R, Ying X, Gao L, Ji Q, Gao Y, Yan Y, et al: Melatonin differentially regulates pathological and physiological cardiac hypertrophy: Crucial role of circadian nuclear receptor ROR $\alpha$ signaling. J Pineal Res 67: e12579, 2019.

3. Shimizu I and Minamino T: Physiological and pathological cardiac hypertrophy. J Mol Cell Cardiol 97: 245-262, 2016.

4. Heineke J and Molkentin JD: Regulation of cardiac hypertrophy by intracellular signalling pathways. Nat Rev Mol Cell Biol 7 : 589-600, 2006.

5. Nakamura M and Sadoshima J: Mechanisms of physiological and pathological cardiac hypertrophy. Nat Rev Cardiol 15: 387-407, 2018.

6. Tsuruda T, Sekita-Hatakeyama Y, Hao Y, Sakamoto S, Kurogi S, Nakamura M, Udagawa N, Funamoto T, Sekimoto T, Hatakeyama K, et al: Angiotensin II stimulation of cardiac hypertrophy and functional decompensation in osteoprotegerin-deficient mice. Hypertension 67: 848-856, 2016.

7. Luo YX, Tang X, An XZ, Xie XM, Chen XF, Zhao X, Hao DL, Chen HZ and Liu DP: SIRT4 accelerates Ang II-induced pathological cardiac hypertrophy by inhibiting manganese superoxide dismutase activity. Eur Heart J 38: 1389-1398, 2017.

8. Liu Y, Jiao R, Ma ZG, Liu W, Wu QQ, Yang Z, Li FF, Yuan Y, Bian ZY and Tang QZ: Sanguinarine inhibits angiotensin II-induced apoptosis in $\mathrm{H} 9 \mathrm{c} 2$ cardiac cells via restoring reactive oxygen species-mediated decreases in the mitochondrial membrane potential. Mol Med Rep 12: 3400-3408, 2015.

9. Murphy E, Ardehali H, Balaban RS, DiLisa F, Dorn GW II, Kitsis RN, Otsu K, Ping P, Rizzuto R, Sack MN, et al: Mitochondrial function, biology, and role in disease: A scientific statement from the American heart association. Circ Res 118: 1960-1991, 2016.

10. Peoples JN, Saraf A, Ghazal N, Pham TT and Kwong JQ: Mitochondrial dysfunction and oxidative stress in heart disease. Exp Mol Med 51: 1-13, 2019.
11. Cai J, Shi G, Zhang Y, Zheng Y, Yang J, Liu Q, Gong Y, Yu D and Zhang Z: Taxifolin ameliorates DEHP-induced cardiomyocyte hypertrophy via attenuating mitochondrial dysfunction and glycometabolism disorder in chicken. Environ Pollut 255: 113155, 2019.

12. Tian H, Yu D, Hu Y, Zhang P, Yang Y, Hu Q and Li M: Angiotensin II upregulates cyclophilin A by enhancing ROS production in rat cardiomyocytes. Mol Med Rep 18: 4349-4355, 2018.

13. Hao P, Jiang F, Cheng J, Ma L, Zhang Y and Zhao Y: Traditional Chinese medicine for cardiovascular disease: Evidence and potential mechanisms. J Am Coll Cardiol 69: 2952-2966, 2017.

14. Gao S, Liu Z, Li H, Little PJ, Liu P and Xu S: Cardiovascular actions and therapeutic potential of tanshinone IIA. Atherosclerosis 220: 3-10, 2012

15. Tan X, Li J, Wang X, Chen N, Cai B, Wang G, Shan H, Dong D, Liu Y, Li X, et al: Tanshinone IIA protects against cardiac hypertrophy via inhibiting calcineurin/NFATc3 pathway. Int J Biol Sci 7: 383-389, 2011.

16. Huang XY and Chen CX: Effect of oxymatrine, the active component from Radix Sophorae flavescentis (Kushen), on ventricular remodeling in spontaneously hypertensive rats. Phytomedicine 20: 202-212, 2013.

17. Liu Y, Liang S, Bu P, Liang E, Yan F, Xing Y and Zhang P: Radix Puerariae rebalances vasomotor factors and improves left ventricular diastolic dysfunction in patients with essential hypertension. Exp Ther Med 20: 705-713, 2020.

18. Wang J, Dong ZH, Gui MT, Yao L, Li JH, Zhou XJ and Fu DY: HuoXue QianYang QuTan recipe attenuates left ventricular hypertrophy in obese hypertensive rats by improving mitochondrial function through SIRT1/PGC-1 $\alpha$ deacetylation pathway. Biosci Rep 39: BSR20192909, 2019.

19. Zhou X, Lu B, Fu D, Gui M, Yao L and Li J: Huoxue Qianyang decoction ameliorates cardiac remodeling in obese spontaneously hypertensive rats in association with ATF6-CHOP endoplasmic reticulum stress signaling pathway regulation. Biomed Pharmacother 121: 109518, 2020.

20. Vaskova E, Ikeda G, Tada Y, Wahlquist C, Mercola M and Yang PC: Sacubitril/valsartan improves cardiac function and decreases myocardial fibrosis via downregulation of exosomal miR-181a in a rodent chronic myocardial infarction model. J Am Heart Assoc 9: e015640, 2020.

21. Ehler E, Moore-Morris T and Lange S: Isolation and culture of neonatal mouse cardiomyocytes. J Vis Exp: 50154, 2013.

22. Xing L and Li Z: Angiotensin II induced myocardial hypertrophy in neonatal rats could be attenuated by activated $\kappa$-opioid receptor via modulating the calcineurin signal pathways. Zhonghua Xin Xue Guan Bing Za Zhi 43: 254-258, 2015 (In Chinese).

23. Livak KJ and Schmittgen TD: Analysis of relative gene expression data using real-time quantitative PCR and the 2(-Delta Delta C(T)) method. Methods 25: 402-408, 2001.

24. Li H, Sureda A, Devkota HP, Pittalà V, Barreca D, Silva AS, Tewari D, Xu S and Nabavi SM: Curcumin, the golden spice in treating cardiovascular diseases. Biotechnol Adv 38: 107343 , 2020.

25. Xiong X, Yang X, Duan L, Liu W, Zhang Y, Liu Y, Wang P, Li S and Li X: Traditional Chinese medicine suppresses left ventricular hypertrophy by targeting extracellular signal-regulated kinases signaling pathway in spontaneously hypertensive rats. Sci Rep 7: 42965, 2017.

26. Huang R, Cui YC, Wei XH, Pan CS, Li Q, He SY, Fan JY and Han JY: A novel traditional Chinese medicine ameliorates fatigue-induced cardiac hypertrophy and dysfunction via regulation of energy metabolism. Am J Physiol Heart Circ Physiol 316: H1378-H1388, 2019.

27. Gao RR, Wu XD, Jiang HM, Zhu YJ, Zhou YL, Zhang HF, Yao WM, Li YQ and Li XL: Traditional Chinese medicine Qiliqiangxin attenuates phenylephrine-induced cardiac hypertrophy via upregulating PPAR $\gamma$ and PGC-1 $\alpha$. Ann Transl Med 6: $153,2018$.

28. Zou R, Tao J, Qiu J, Shi W, Zou M, Chen W, Li W, Zhou N, Wang S, Ma L and Chen X: Ndufs1 deficiency aggravates the mitochondrial membrane potential dysfunction in pressure overload-induced myocardial hypertrophy. Oxid Med Cell Longev 2021: 5545261, 2021.

29. Thirupathi A and de Souza CT: Multi-regulatory network of ROS: The interconnection of ROS, PGC-1 alpha, and AMPK-SIRT1 during exercise. J Physiol Biochem 73: 487-494, 2017.

30. St-Pierre J, Drori S, Uldry M, Silvaggi JM, Rhee J, Jäger S, Handschin C, Zheng K, Lin J, Yang W, et al: Suppression of reactive oxygen species and neurodegeneration by the PGC-1 transcriptional coactivators. Cell 127: 397-408, 2006. 
31. Rababa'h AM, Guillory AN, Mustafa R and Hijjawi T: Oxidative stress and cardiac remodeling: An updated edge. Curr Cardiol Rev 14: 53-59, 2018.

32. Gallo S, Vitacolonna A, Bonzano A, Comoglio P and Crepaldi T: ERK: A key player in the pathophysiology of cardiac hypertrophy. Int J Mol Sci 20: 2164, 2019.

33. Singh RM, Cummings E, Pantos C and Singh J: Protein kinase C and cardiac dysfunction: A review. Heart Fail Rev 22: 843-859, 2017.

34. Lee Y, Jeong GS, Kim KM, Lee W and Bae JS: Cudratricusxanthone A attenuates sepsis-induced liver injury via SIRT1 signaling. J Cell Physiol 233: 5441-5446, 2018.

35. Cui L, Guo J, Zhang Q, Yin J, Li J, Zhou W, Zhang T, Yuan H, Zhao J, Zhang L, et al: Erythropoietin activates SIRT1 to protect human cardiomyocytes against doxorubicin-induced mitochondrial dysfunction and toxicity. Toxicol Lett 275: $28-38,2017$.
36. Zhang T, Chi Y, Ren Y, Du C, Shi Y and Li Y: Resveratrol reduces oxidative stress and apoptosis in podocytes via Sir2-related enzymes, sirtuins1 (SIRT1)/peroxisome proliferator-activated receptor $\gamma$ co-activator $1 \alpha(\mathrm{PGC}-1 \alpha)$ axis. Med Sci Monit 25: 1220-1231, 2019.

This work is licensed under a Creative Commons Attribution-NonCommercial-NoDerivatives 4.0 International (CC BY-NC-ND 4.0) License. 